\title{
Efeitos do consumo de drogas parental no desenvolvimento e saúde mental da criança: revisão integrativa
}

\author{
Lany Leide de Castro Rocha Campelo ${ }^{1}$ \\ Raionara Cristina de Araujo Santos ${ }^{2}$ \\ Margareth Angelo ${ }^{3}$ \\ Maria do Perpétuo Socorro de Sousa Nóbrega ${ }^{3}$
}

\begin{abstract}
Objetivo: analisar as publicações que abordam o impacto do consumo de drogas parental no desenvolvimento e na saúde mental das crianças. Método: revisão integrativa na Biblioteca Virtual em Saúde e no PUBMED, utilizando-se os descritores "Child development", "Mental health", "Parents", "Mothers", "Drug users" e "Child". Resultados: a amostra final totalizou 11 artigos sobre efeitos neonatais do consumo de drogas em gestantes e consequências do uso de drogas no desenvolvimento cognitivo, motor e psicossocial das crianças. Conclusão: o consumo de drogas parental compromete o crescimento e o desenvolvimento da criança, podendo ocasionar problemas comportamentais e de saúde mental, seja pelas consequências diretas ou pelos efeitos indiretos do consumo dessas substâncias na criança.
\end{abstract}

Descritores: Desenvolvimento Infantil; Saúde Mental; Pais; Usuários de Drogas.

\footnotetext{
1 Universidade Federal do Piauí, Picos, PI, Brasil.

2 Universidade Federal do Rio Grande do Norte, Departamento de Enfermagem, Rio Grande do Norte, RN, Brasil.

${ }^{3}$ Universidade de São Paulo, Escola de Enfermagem, São Paulo, SP, Brasil.
} 


\section{Effects of parental drug use on child development and mental health:} integrative review

Objective: to analyze publications dealing with the impact of drug use by parents on the development and mental health of children. Method: comprehensive Review in the Virtual Health Library and PUBMED, using the descriptors "Child development", "Mental health", "Parents", "Mothers", "Drug users" and "Child". Results: the final sample totaled 11 articles on: neonatal effects of drug use by pregnant women and consequences of drug use on the cognitive, motor and psychosocial development of children. Conclusion: drug use by parents jeopardizes the child's growth and development and can lead to behavioral and mental health problems, either because of direct consequences or because of indirect effects of the child's consumption of these substances.

Descriptors: Child Development; Mental Health; Parents; Drug Users.

\section{Efectos del consumo de drogas por los padres en el desarrollo y}

\section{la salud mental de los niños: revisión integrativa}

Objectivo: analizar en publicaciones el impacto del consumo de drogas por los padres en el desarrollo y la salud mental de los niños. Método: revisión integradora en la Biblioteca Virtual en Salud y la PubMed, utilizando los descriptores "Child development", "Mental health", "Parents", "Mothers", "Drug users" and "Child". Resultados: la muestra final de 11 artículos sobre: efectos neonatales del consumo de drogas en las mujeres embarazadas y sobre las consecuencias del consumo de drogas en el desarrollo cognitivo, motor y psicosociales de los niños. Conclusión: el consumo de drogas por los padres compromete el crecimiento y desarrollo de los niños, y puede causar problemas de comportamiento y de salud mental, sea por la consecuencia directa o por efectos indirectos del consumo en la criança.

Descriptores: Desarrollo Infantil; Salud Mental; Padres; Usuarios de Drogas.

\section{Introdução}

O desenvolvimento infantil é permeado por inúmeros condicionantes, incluindo desde fatores genéticos e orgânicos, relacionados com características individuais do ser humano em formação, a fatores mais amplos, como influências ambientais, culturais e sociais, que se manifestam, por exemplo, a partir da família, escola e sociedade ${ }^{(1)}$.

Na literatura disponível existem diversas pesquisas cujo objetivo consiste em investigar eventos que podem influenciar o desenvolvimento e a saúde mental de crianças a partir de diversos enfoques, tais como fatores intrínsecos presentes nas crianças ${ }^{(2)}$, influência de características parentais ${ }^{(3)}$ ou de situações ambientais $^{(3-4)}$.

Crianças e adolescentes com deficit no desenvolvimento podem ter sérios prejuízos em seu desempenho funcional e em sua saúde mental, que vão desde a diminuição na capacidade de realizar atividades rotineiras do cotidiano de maneira satisfatória e apropriada para cada etapa do desenvolvimento até tornarem-se alvos de ações de bullying, violência, preconceito, entre outros.

As características parentais, assim como os fatores ambientais, constituem-se tanto em fatores de risco 
como de proteção para o desenvolvimento e saúde mental das crianças ${ }^{(5)}$, podendo afetá-las de forma independente.

Considera-se fator de proteção as condições que permitem o fortalecimento, enfrentamento, crescimento e desenvolvimento pleno do ser em formação. Por sua vez, os fatores de risco são variáveis ou condições que interferem no bem-estar, na saúde ou no desempenho social de um indivíduo ou de um grupo social, ocasionando resultados negativos ao seu desenvolvimento(6)

Entre os fatores de risco, o uso de drogas parental e seu impacto sobre o desenvolvimento e a saúde mental de crianças têm sido amplamente pesquisados e relatados em periódicos nacionais e internacionais ${ }^{(7-8)}$. A alta prevalência e os diversos problemas associados ao abuso drogas parental inserem esse tema como uma relevante questão de saúde pública e chama a atenção para a necessidade de intervenções diante dessa realidade(8).

A exposição ao álcool e outras drogas é uma das muitas influências negativas do meio ambiente para o desenvolvimento infantil. Em muitos casos, o uso parental da substância é um marcador substancial para a disfunção familiar levando a resultados previsivelmente negativos para as crianças ${ }^{(7)}$. Somado a isso, crianças nascidas de gestações desfavoráveis e vindas de situação socioeconômica adversa são expostas a vários riscos e a uma maior tendência a atrasos no seu desenvolvimento neuropsicomotor ${ }^{(9)}$.

Estudo tem apontado que o uso de substâncias psicoativas por mulheres grávidas está relacionado a prejuízos nas funções cognitivas das crianças, impactando em dificuldades na manutenção da atenção e prejuízos na memória e no aprendizado, com maior deficiência ou retardo no desenvolvimento cognitivo em crianças de até dois anos(7).

Não obstante, apesar de não se poder definir as doses tóxicas, o tempo de uso prejudicial, tampouco determinar um padrão específico de problemas causados por cada droga especificamente, o abuso de drogas em geral está associado a um maior risco de anomalias na gravidez pelo incremento do risco tanto de malformações congênitas quanto da morbidade e mortalidade perinatal desencadeadas por deficit variáveis nas medidas antropométricas de nascimento, desconforto respiratório, infecção neonatal, icterícia, edema agudo de pulmão, sífilis congênita e sofrimento, por exemplo(10-12).

Além disso, foram descritas alterações neurocomportamentais transitórias de leve a moderada na infância, problemas comportamentais em longo prazo observados desde a infância até a adolescência, assim como possíveis repercussões em longo prazo na capacidade de aprendizagem de crianças expostas intraútero ${ }^{(12)}$, sendo a gravidade da expressão de tais problemas influenciada por fatores ambientais ${ }^{(11)} e$ pela extensão do período de exposição do feto às substâncias ${ }^{(10)}$.

Diante do exposto, este estudo objetivou analisar as publicações nacionais e internacionais que abordam o impacto do consumo de drogas parental no desenvolvimento e na saúde mental das crianças.

\section{Método}

Trata-se de uma revisão integrativa, a qual proporciona a síntese de conhecimentos relevantes que dão suporte para a tomada de decisão e melhoria da prática clínica, com a incorporação de resultados de estudos significativos para a prática e apontamento das lacunas no conhecimento sobre determinado assunto(13).

Estabeleceram-se as seguintes etapas a serem cumpridas para a execução deste estudo: identificação do problema e definição da questão norteadora; definição dos critérios de inclusão e exclusão dos artigos; seleção das bases de dados e busca das produções científicas; escolha dos artigos e análise dos estudos incluídos; desenvolvimento da discussão e síntese da revisão(14).

Para guiar a revisão integrativa, elaborou-se a seguinte questão norteadora: Qual(is) o(s) efeito(s) do consumo de drogas parental no desenvolvimento e na saúde mental da criança?

Realizou-se a seleção dos artigos de forma independente, por duas autoras, no mês de março de 2015. Todos os resultados obtidos foram comparados entre si para verificar a concordância dos dados obtidos.

Utilizou-se a Biblioteca Virtual em Saúde (BVS) por englobar várias bases de dados nacionais e algumas internacionais, como também a base de dados PUBMED. Desse modo, procurou-se ampliar o âmbito da pesquisa minimizando possíveis vieses nessa etapa do processo de elaboração da revisão integrativa.

Fez-se a busca dos artigos por meio do cruzamento dos Descritores em Ciências da Saúde no idioma inglês: Child development, Mental health, Parents, Mothers, Drug users e Child, empregando-se os operadores booleanos AND e OR, em combinações que totalizaram 10 estratégias de busca diferentes, conforme apresentados na Tabela 1.

Incluíram-se artigos primários publicados nos últimos 10 anos (de 2005 a 2015); em inglês, português e espanhol; que retrataram o desenvolvimento e/ou a saúde mental de crianças de zero a 12 anos, filhos de pais e/ou mães que usavam ou abusavam de 
drogas (lícitas e/ou ilícitas); excepcionalmente foram mantidos nesta revisão dois artigos que incluíram crianças com idade acima de 12 anos por terem sido realizados as que se apresentavam na faixa etária desta revisão. Excluíram-se os artigos cuja abordagem central tratava da presença de doenças como HIV e hepatite, que podem contribuir para aumentar a vulnerabilidade infantil; artigos cujos textos completos não foram disponibilizados na íntegra; artigos sobre o desenvolvimento e/ou a saúde mental que não incluíam crianças de 0 a 12 anos de idade; e artigos de revisão.

A busca inicial encontrou 188 artigos na BVS e 1310 no PUBMED. Aplicando-se os critérios de artigos primários publicados nos últimos 10 anos, em inglês, português e espanhol, esse total reduziu-se para 99 artigos na BVS e 516 no PUBMED.
Em seguida, procedeu-se à leitura dos títulos e resumos desses artigos, selecionando-se aqueles que retratassem o desenvolvimento e/ou a saúde mental de crianças com idade de 0 a 12 anos, filhos de pais e/ou mães usuários de drogas (lícitas e/ou ilícitas), totalizando 20 artigos na BVS e 64 no PUBMED.

Após eliminarem-se as repetições de artigos existentes na BVS e na base de dados PUBMED, bem como em todas as estratégias de busca utilizadas, obteve-se o total de 21 artigos para a leitura. Destes, ainda se excluíram um artigo por ser de revisão, seis por não abordarem a temática do estudo, um por ausência do texto completo, inviabilizando sua análise, e um por ter sido realizado apenas com adultos filhos de mães usuárias de drogas, totalizando uma amostra final de 11 artigos.

Tabela 1 - Estratégia de busca utilizada. Ribeirão Preto, SP, Brasil, 2015

\begin{tabular}{|c|c|c|c|c|c|c|}
\hline Estratégia de busca & BVS & Filtros & PUBMED & Filtros & Selecionados & Repetidos \\
\hline $\begin{array}{c}\text { Child development AND Mental } \\
\text { health AND Parents OR Mothers } \\
\text { AND Drug users }\end{array}$ & 1 & 0 & 113 & 11 & 11 & 0 \\
\hline $\begin{array}{l}\text { Child development OR Mental } \\
\text { health AND Parents OR Mothers } \\
\text { AND Drug users }\end{array}$ & 19 & 3 & 129 & 11 & 14 & 7 \\
\hline $\begin{array}{l}\text { Child development AND Mental } \\
\text { health AND Parents AND Drug } \\
\text { users }\end{array}$ & 2 & 0 & 2 & 0 & 0 & 0 \\
\hline $\begin{array}{c}\text { Child development AND Mental } \\
\text { health AND Mothers AND Drug } \\
\text { users }\end{array}$ & 1 & 0 & 4 & 1 & 1 & 1 \\
\hline $\begin{array}{l}\text { Child development AND Parents } \\
\text { OR Mothers AND Drug users }\end{array}$ & 13 & 5 & 117 & 16 & 21 & 20 \\
\hline $\begin{array}{l}\text { Child development AND Parents } \\
\text { AND Drug users }\end{array}$ & 8 & 1 & 15 & 2 & 3 & 3 \\
\hline $\begin{array}{l}\text { Child development AND Mothers } \\
\text { AND Drug users }\end{array}$ & 13 & 3 & 21 & 3 & 6 & 6 \\
\hline $\begin{array}{l}\text { Child AND Mental health AND } \\
\text { Parents OR Mothers AND Drug } \\
\text { users }\end{array}$ & 11 & 3 & 118 & 17 & 20 & 18 \\
\hline $\begin{array}{l}\text { Child AND Mental health AND } \\
\text { Parents AND Drug users }\end{array}$ & 20 & 2 & 9 & 1 & 3 & 3 \\
\hline $\begin{array}{l}\text { Child AND Mental health AND } \\
\text { Mothers AND Drug users }\end{array}$ & 11 & 3 & 8 & 2 & 5 & 5 \\
\hline TOTAL & 99 & 20 & 531 & 64 & 84 & 63 \\
\hline
\end{tabular}

Para a análise e posterior síntese dos 11 artigos selecionados, utilizou-se um quadro sinóptico, especialmente construído para esse fim, que contemplou: referência do artigo, país do estudo, objetivo, tipo de estudo, amostra, principais resultados e principais conclusões.

\section{Resultados}

Os estudos foram publicados predominantemente no período de 2009 a 2014, com apenas um estudo publicado em $2005^{(15)}$ e outro em $2007^{(16)}$. Quanto à origem e idioma das publicações, dez artigos foram 
publicados em inglês, sendo dois provenientes do Reino Unido(17-18), dois na Noruega ${ }^{(19-20)}$, dois na Austrália ${ }^{(21-22)}$, dois nos EUA ${ }^{(16,23)}$, um na Tailândia(15) e um no Canadá(24), e um único artigo publicado em espanhol, na Espanha(25).

Com relação ao tipo de estudo, identificou-se um predomínio de pesquisas quantitativas, das quais sete foram do tipo coorte retrospectivo ${ }^{(15,18,20-22,24-25)}$, recuperando informações das crianças desde o seu nascimento até a alta hospitalar; uma de coorte prospectivo $^{(17)}$, acompanhando o desenvolvimento da criança até os 12 meses de idade; dois estudos comparativos longitudinais, um com seguimento de crianças do nascimento aos 2 anos de idade ${ }^{(23)}$ e outro do nascimento até os 13 anos de idade ${ }^{(19)}$; e um estudo transversal que inclui crianças e adolescentes de 12 a 17 anos de idade ${ }^{(16)}$. Os resultados da síntese dos artigos selecionados foram apresentados na Figura 1.

\begin{tabular}{|c|c|c|c|c|c|}
\hline Autor (Ano) & País & Objetivo & $\begin{array}{l}\text { Tipo de } \\
\text { estudo }\end{array}$ & Amostra & Principais resultados \\
\hline $\begin{array}{l}\text { Singer et al. } \\
(2012)^{(17)} \text {. }\end{array}$ & $\begin{array}{l}\text { Reino } \\
\text { Unido }\end{array}$ & $\begin{array}{l}\text { Apresentar } \\
\text { resultados de follow- } \\
\text { up de } 12 \text { meses } \\
\text { de uma coorte de } \\
\text { crianças expostas ao } \\
\text { ecstasy no pré-natal. }\end{array}$ & $\begin{array}{c}\text { Coorte } \\
\text { prospectivo }\end{array}$ & $\begin{array}{l}96 \text { mulheres } \\
\text { (28 usuárias de } \\
\text { ecstasy durante } \\
\text { a gravidez e } 68 \\
\text { não usuárias) e } 79 \\
\text { crianças de até } 1 \\
\text { ano de idade. }\end{array}$ & $\begin{array}{l}\text { O acompanhamento das } \\
\text { crianças por } 12 \text { meses mostrou } \\
\text { que o uso de ecstasy na } \\
\text { gestação impactou em efeitos } \\
\text { negativos sobre os resultados } \\
\text { cognitivos e motores das } \\
\text { crianças, sendo maior neste } \\
\text { último. }\end{array}$ \\
\hline $\begin{array}{l}\text { Gomes et al. } \\
(2011)^{(25)}\end{array}$ & Espanha & $\begin{array}{l}\text { Conhecer a } \\
\text { prevalência atual, } \\
\text { as características } \\
\text { da gravidez, parto y } \\
\text { recém- nascidos de } \\
\text { mães consumidoras } \\
\text { de drogas. }\end{array}$ & $\begin{array}{l}\text { Coorte } \\
\text { retrospectivo }\end{array}$ & $\begin{array}{c}157 \text { recém- } \\
\text { nascidos de mães } \\
\text { consumidoras } \\
\text { de alguma } \\
\text { droga (cocaína, } \\
\text { heroína, cannabis, } \\
\text { benzodiazepinas } \\
\text { e anfetaminas, } \\
\text { metadona). }\end{array}$ & $\begin{array}{l}\text { O consumo de drogas pelas } \\
\text { mães esteve associado à } \\
\text { prematuridade, presença } \\
\text { de síndrome de abstinência } \\
\text { de drogas, alteração na } \\
\text { sucção, tremor, irritabilidade } \\
\text { e hiperreflexia nos recém- } \\
\text { nascidos. }\end{array}$ \\
\hline $\begin{array}{l}\text { Hjerkinn et al. } \\
(2013)^{(19)} \text {. }\end{array}$ & Noruega & $\begin{array}{l}\text { Investigar o } \\
\text { comportamento } \\
\text { de crianças filhas de } \\
\text { mães usuárias de } \\
\text { drogas e relacioná-lo } \\
\text { com o seu cuidador } \\
\text { (mães biológicas } \\
\text { ou pais adotivos) } \\
\text { e correlacionar os } \\
\text { resultados dos testes } \\
\text { de comportamento } \\
\text { das crianças com } \\
\text { os da avaliação } \\
\text { neuropsicológica. }\end{array}$ & $\begin{array}{c}\text { Quasi- } \\
\text { experimental, } \\
\text { longitudinal }\end{array}$ & $\begin{array}{l}38 \text { recém-nascidos } \\
\text { de mães usuárias } \\
\text { de substâncias } \\
\text { psicoativas } \\
\text { (cannabis e } \\
\text { metanfetaminas) } \\
\text { durante a gravidez, } \\
\text { comparadas com } \\
80 \text { crianças, filhas } \\
\text { de mães não } \\
\text { usuárias dessas } \\
\text { substâncias. }\end{array}$ & $\begin{array}{l}\text { Filhos de mulheres que abusam } \\
\text { de substâncias apresentaram } \\
\text { mais problemas acadêmicos, } \\
\text { precisaram de mais atendimento } \\
\text { corretivo na escola, foram mais } \\
\text { frequentemente diagnosticados } \\
\text { com transtorno de deficit } \\
\text { de atenção/hiperatividade, } \\
\text { depressão, transtorno de } \\
\text { ansiedade ou distúrbio somático }\end{array}$ \\
\hline $\begin{array}{l}\text { Hjerkinn et al. } \\
\qquad(2009)^{(20)} \text {. }\end{array}$ & Noruega & $\begin{array}{l}\text { Comparar os } \\
\text { resultados neonatais } \\
\text { das crianças filhas } \\
\text { de mães que } \\
\text { abusaram de drogas } \\
\text { atendidas em uma } \\
\text { clínica de bem-estar } \\
\text { para crianças com } \\
\text { crianças filhas de } \\
\text { mães que não eram } \\
\text { toxicodependentes. }\end{array}$ & $\begin{array}{c}\text { Coorte } \\
\text { retrospectivo }\end{array}$ & $\begin{array}{c}62 \text { recém- } \\
\text { nascidos de } 59 \\
\text { mães usuárias } \\
\text { de anfetaminas } \\
\text { e opiáceos, } \\
\text { comparados com } \\
\text { filhos de mães } \\
\text { não usuárias de } \\
\text { drogas. }\end{array}$ & $\begin{array}{l}\text { O grupo de filhos de mães } \\
\text { usuárias de drogas apresentou } \\
\text { maior taxa de prematuridade, } \\
\text { baixo peso e menor perímetro } \\
\text { cefálico ao nascer, quando } \\
\text { comparado ao grupo de filhos de } \\
\text { mães não usuárias de drogas. } \\
\text { O filhos de mães que param } \\
\text { de consumir drogas no início } \\
\text { da gravidez tenderam a ter } \\
\text { resultados de nascimento } \\
\text { semelhantes aos das mães sem } \\
\text { abuso de substâncias. }\end{array}$ \\
\hline
\end{tabular}

A Figura 1 continua na próxima página... 


\begin{tabular}{|c|c|c|c|c|c|}
\hline Autor (Ano) & País & Objetivo & $\begin{array}{l}\text { Tipo de } \\
\text { estudo }\end{array}$ & Amostra & Principais resultados \\
\hline $\begin{array}{c}\text { Richardson } \\
\text { et al. } \\
(2011)^{(23)} \text {. }\end{array}$ & EUA & $\begin{array}{l}\text { Abordar as relações } \\
\text { entre consumo de } \\
\text { cocaína pré-natal } \\
\text { e desenvolvimento } \\
\text { físico, cognitivo e } \\
\text { comportamental de } \\
\text { crianças. }\end{array}$ & $\begin{array}{c}\text { Estudo } \\
\text { comparativo, } \\
\text { longitudinal }\end{array}$ & $\begin{array}{c}243 \text { mães usuárias } \\
\text { de cocaína e/ } \\
\text { ou crack e } \\
\text { suas crianças } \\
\text { (examinadas } \\
\text { ao nascer e, } \\
\text { posteriormente, } \\
\text { com } 1,3 \text { e } 7 \text { anos } \\
\text { de idade). }\end{array}$ & $\begin{array}{c}\text { As crianças expostas à } \\
\text { cocaína durante a gravidez } \\
\text { apresentaram mais problemas } \\
\text { de comportamento avaliado por } \\
\text { mães e professores. }\end{array}$ \\
\hline $\begin{array}{c}\text { Blandthorn } \\
\text { et al. } \\
(2011)^{(21)} \text {. }\end{array}$ & Austrália & $\begin{array}{l}\text { Descrever as } \\
\text { características } \\
\text { maternas, da } \\
\text { gravidez e os } \\
\text { resultados } \\
\text { neonatais em um } \\
\text { grupo de mulheres } \\
\text { grávidas, usuárias } \\
\text { de substancia } \\
\text { psicoativa, em } \\
\text { tratamento } \\
\text { farmacológico de } \\
\text { substituição com } \\
\text { buprenorfina ou } \\
\text { metadona. }\end{array}$ & $\begin{array}{c}\text { Estudo } \\
\text { descritivo } \\
\text { retrospectivo }\end{array}$ & $\begin{array}{l}98 \text { recém-nascidos } \\
\text { e suas mães } \\
\text { usuárias de tabaco, } \\
\text { cannabis, heroína, } \\
\text { benzodiazepínicos, } \\
\text { álcool, anfetaminas } \\
\text { e/ou morfina em } \\
\text { tratamento com } \\
\text { metadona ou } \\
\text { buprenorfina. }\end{array}$ & $\begin{array}{l}\text { Observou-se uma proporção } \\
\text { significativa de nascimentos } \\
\text { prematuros e baixo peso. } \\
\text { Algumas crianças nascidas } \\
\text { com menos de } 33 \text { semanas de } \\
\text { gestação receberam medicação } \\
\text { para tratamento de síndrome de } \\
\text { abstinência neonatal. }\end{array}$ \\
\hline $\begin{array}{l}\text { Dryden et al. } \\
(2009)^{(18)} \text {. }\end{array}$ & $\begin{array}{l}\text { Reino } \\
\text { Unido }\end{array}$ & $\begin{array}{l}\text { Investigar os fatores } \\
\text { associados ao } \\
\text { desenvolvimento } \\
\text { da síndrome de } \\
\text { abstinência neonatal } \\
\text { e avaliar as } \\
\text { implicações em } \\
\text { crianças nascidas } \\
\text { de mulheres em uso } \\
\text { abusivo de drogas } \\
\text { para os recursos de } \\
\text { saúde. }\end{array}$ & $\begin{array}{l}\text { Coorte } \\
\text { retrospectivo }\end{array}$ & $\begin{array}{c}437 \text { recém- } \\
\text { nascidos e } 440 \\
\text { mães usuárias } \\
\text { de substâncias } \\
\text { psicoativas } \\
\text { (benzodiazepina, } \\
\text { heroína, cannabis, } \\
\text { cocaína, } \\
\text { anfetamina, álcool } \\
\text { e/ou tabaco) } \\
\text { durante a gestação } \\
\text { e em tratamento } \\
\text { com metadona. }\end{array}$ & $\begin{array}{l}\text { Observou-se uma parcela } \\
\text { considerável de recém-nascidos } \\
\text { prematuros, com problemas } \\
\text { respiratórios, baixo peso e } \\
\text { perímetro cefálico menor que } \\
\text { a média. O uso materno de } \\
\text { benzodiazepínicos em adição } \\
\text { ao tratamento com metadona } \\
\text { aumentou significativamente a } \\
\text { probabilidade de aparecimento } \\
\text { de síndrome de abstinência } \\
\text { neonatal e a necessidade de } \\
\text { tratamento farmacológico. }\end{array}$ \\
\hline $\begin{array}{l}\text { Pong et al. } \\
(2010)^{(22)} \text {. }\end{array}$ & Austrália & $\begin{array}{l}\text { Determinar se } \\
\text { a exposição a } \\
\text { drogas e resultados } \\
\text { perinatais são } \\
\text { afetados por } \\
\text { mudanças na } \\
\text { disponibilidade de } \\
\text { drogas na rua. }\end{array}$ & $\begin{array}{l}\text { Coorte } \\
\text { retrospectivo }\end{array}$ & $\begin{array}{c}293 \\
\text { mães dependentes } \\
\text { de drogas } \\
\text { (metadona, } \\
\text { heroína, } \\
\text { benzodiazepínicos, } \\
\text { cocaína, } \\
\text { anfetaminas, } \\
\text { cigarro, álcool e } \\
\text { cannabis) e } 297 \\
\text { lactentes. }\end{array}$ & $\begin{array}{l}\text { As crianças que foram expostas } \\
\text { a vários tipos de drogas } \\
\text { intraútero permaneceram } \\
\text { hospitalizadas por mais tempo } \\
\text { em comparação com as crianças } \\
\text { expostas a uma única } \\
\text { classe de drogas. } \\
\text { Uma parcela significativa dos } \\
\text { recém-nascidos necessitou } \\
\text { de medicamentos para tratar } \\
\text { síndrome de abstinência } \\
\text { neonatal. }\end{array}$ \\
\hline
\end{tabular}

A Figura 1 continua na próxima página... 


\begin{tabular}{|c|c|c|c|c|c|}
\hline Autor (Ano) & País & Objetivo & $\begin{array}{l}\text { Tipo de } \\
\text { estudo }\end{array}$ & Amostra & Principais resultados \\
\hline $\begin{array}{c}\text { Thaithumyano } \\
\text { et al. } \\
(2005)^{(15)}\end{array}$ & Tailândia & $\begin{array}{c}\text { Determinar os } \\
\text { impactos perinatais } \\
\text { de heroína e } \\
\text { anfetamina em mães } \\
\text { e bebês. }\end{array}$ & $\begin{array}{c}\text { Estudo } \\
\text { comparativo } \\
\text { e } \\
\text { retrospectivo }\end{array}$ & $\begin{array}{l}211 \text { mães usuárias } \\
\text { de drogas } \\
\text { (anfetaminas ou } \\
\text { heroína) e } 211 \\
\text { recém-nascidos. }\end{array}$ & $\begin{array}{l}\text { A incidência de prematuridade, } \\
\text { baixo peso ao nascer e } \\
\text { microcefalia não foram } \\
\text { estatisticamente diferentes entre } \\
\text { os dois grupos de crianças } \\
\text { (expostas à anfetamina e } \\
\text { expostas à heroína). } \\
\text { Uma parcela significativa dos } \\
\text { bebês expostos à heroína } \\
\text { desenvolveu sintomas de } \\
\text { abstinência neonatal. }\end{array}$ \\
\hline $\begin{array}{l}\text { Kelly et al. } \\
(2011)^{(24)} \text {. }\end{array}$ & Canadá & $\begin{array}{l}\text { Documentar a } \\
\text { incidência de } \\
\text { síndrome de } \\
\text { abstinência neonatal } \\
\text { e a taxa de uso de } \\
\text { narcóticos durante } \\
\text { a gravidez, no } \\
\text { noroeste de Ontário. }\end{array}$ & $\begin{array}{l}\text { Estudo } \\
\text { descritivo } \\
\text { retrospectivo }\end{array}$ & $\begin{array}{l}61 \text { mães usuárias } \\
\text { de narcóticos } \\
\text { (oxicodona) e } 61 \\
\text { recém-nascidos. }\end{array}$ & $\begin{array}{l}\text { Os filhos de mães usuárias de } \\
\text { drogas apresentaram maiores } \\
\text { taxas de prematuridade, alguns } \\
\text { exibiram sintomas de síndrome } \\
\text { de abstinência neonatal, sendo } \\
\text { o uso diário de drogas pela } \\
\text { mãe um fator agravante do } \\
\text { surgimento da síndrome. }\end{array}$ \\
\hline $\begin{array}{l}\text { Lam et al. } \\
(2007)^{(16)} \text {. }\end{array}$ & EUA & $\begin{array}{c}\text { Analisar as } \\
\text { relações entre } \\
\text { comportamentos } \\
\text { parentais, qualidade } \\
\text { do vínculo e efeitos } \\
\text { moderadores } \\
\text { da idade sobre } \\
\text { o consumo de } \\
\text { substâncias pelos } \\
\text { jovens em uma } \\
\text { amostra de uma } \\
\text { comunidade } \\
\text { de mães afro- } \\
\text { americanas que } \\
\text { usam cocaína e } \\
\text { crack e seus filhos } \\
\text { de } 12 \text { a } 17 \text { anos. }\end{array}$ & $\begin{array}{c}\text { Estudo } \\
\text { transversal }\end{array}$ & $\begin{array}{l}208 \text { mães usuárias } \\
\text { de crack, cocaína, } \\
\text { álcool e maconha } \\
\text { e } 208 \text { crianças e } \\
\text { adolescentes com } \\
\text { idades entre } 12 \text { e } \\
17 \text { anos. }\end{array}$ & $\begin{array}{l}\text { O efeito de conflitos familiares } \\
\text { sobre o uso de substâncias } \\
\text { nas crianças e adolescentes } \\
\text { variou consoante a idade. Entre } \\
\text { os mais velhos, aqueles que } \\
\text { perceberam um alto nível de } \\
\text { conflitos familiares foram mais } \\
\text { propensos a usar substâncias } \\
\text { em comparação com aqueles } \\
\text { que perceberam um menor nível } \\
\text { de conflito familiar. Entre os mais } \\
\text { velhos, a desaprovação materna } \\
\text { foi proteção contra o uso de } \\
\text { substâncias. }\end{array}$ \\
\hline
\end{tabular}

Figura 1 - Síntese dos artigos selecionados. Ribeirão Preto, SP, Brasil, 2015

A maior parte dos artigos preocupou-se em estudar os efeitos neonatais do consumo de drogas em gestantes ${ }^{(15,18,20-22,24-25)}$, correlacionando o uso dessas substâncias com alterações no desenvolvimento físico, tais como baixo peso ao nascer e menor perímetro cefálico ${ }^{(20,23)}$, prematuridade ${ }^{(20,24)}$, baixo Apgar ${ }^{(24-25)}$, pequenos para a idade gestacional(21), além de necessidades de cuidados especiais em decorrência da síndrome de dificuldade respiratória ${ }^{(21)}$, icterícia exigindo fototerapia ${ }^{(21)}$, síndrome de abstinência neonatal|(15,18,21-22), malformações congênitas e morte ${ }^{(15)}$. Esses estudos trouxeram informações sobre o período gestacional, como o número de consultas pré-natais, contando como insuficientes, um número de consultas $<4^{(25)} \mathrm{e}<5^{(22)}$, tipo de parto, complicações na gravidez, como ruptura prematura de membranas e descolamento prematuro da placenta ${ }^{(25)}$ e uso de substâncias durante a gestação.

Um número menor de estudos ${ }^{(16-17,19,23)}$ avaliou as consequências do uso de drogas pela mãe sobre o desenvolvimento cognitivo, motor e psicossocial das crianças, revelando a sua associação com problemas acadêmicos, necessidade de maior suporte ou reforço na escola, e diagnóstico mais frequente de Transtorno de Deficit de Atenção e Hiperatividade (TDAH), depressão ou transtorno de ansiedade ou distúrbio somático(19); uso de drogas associado a variáveis parentais, como conflito familiar, vínculo entre a mãe e a criança e desaprovação materna do uso de substâncias pela criança consoante a idade e a percepção dessas variáveis pela criança( ${ }^{(16)}$; efeitos negativos sobre os 
resultados cognitivos e motores infantis, tais como alterações neurocomportamentais ${ }^{(25)}$, sugerindo risco de desenvolvimento significativo para a criança mais fortemente exposta à droga, como atrasos em ficar de pé e caminhar, menor orientação e engajamento(17).

Com relação aos termos utilizados para definir o tipo de consumo realizado pelas mães, 4 artigos utilizaram o termo "uso de drogas"(16-17,22,25), 1 artigo o termo "uso frequente"(23), 5 artigos o termo "abuso de drogas" $"(18-21,24)$ e 1 artigo o termo "viciadas"(15), levando em conta o autorrelato da mãe, seu histórico médico, a determinação de substâncias tóxicas na urina da mãe ou do RN ou a inclusão da mãe em programas de tratamento.

Os dados, em sua maioria, foram obtidos de prontuários de clínicas ou hospitais que prestam cuidados a gestantes usuárias de drogas, a partir de registros sobre o uso de drogas informado, características demográficas das gestantes; dados sobre a gestação; número de consultas pré-natais; número de partos e nascimentos; registros de ambulatório pediátrico e resultados relacionados ao tempo de permanência no hospital e alta do bebê $\hat{e}^{(15,18,21-22,24-25)}$. Em um estudo, os pais receberam um questionário na reunião da escola ou da clínica, no qual deveriam responder sobre a gravidez, parto, nascimento e evolução da criança, abuso de substâncias, saúde da mãe e da criança, condições de vida e nível educacional(20).

Um menor número de estudos entrevistou as mães sobre 0 uso de substâncias psicoativas ${ }^{(17,24)}$. Para avaliar o comportamento das crianças, utilizaram-se escalas e entrevistas com as mães/pais, professores e crianças ${ }^{(16-17,19,23)}$. O Child Behavior Checklist $(\mathrm{CBCL})$ foi utilizado em um estudo(19). Para avaliação neuropsicológica das crianças, um estudo utilizou a terceira versão do Wechsler Intelligence Scale for Children (WISC-III), NEPSY, Halstead- Reitan and Raven's Progressive Matrices ${ }^{(19)}$.

Foi preocupação de todos os estudos descrever em maior ou menor grau as características socioeconômicas e demográficas das mães, tais como idade, renda/ empregabilidade, nível educacional, região onde vivem, estado civil ou presença do companheiro. Nos estudos em que foi possível identificar a faixa etária das mães, a média de idades variou entre $28^{(18,25)} \mathrm{e}$ 36,8 anos $^{(16)}$. Em quatro estudos, a maioria das mães trabalhava ${ }^{(16-17,23,25)}$; nos demais, a maioria se mantinha com benefícios sociais ${ }^{(18,20,22)}$. O nível educacional apresentou uma grande variação, indo desde pouca ou nenhuma educação escolar ${ }^{(20)}$ até mães com formação universitária ${ }^{(17)}$. Com exceção de um estudo(17), em todos os demais prevaleceu um número maior de mães solteiras, sem companheiro.
Dois estudos identificaram a presença de violência contra mulheres. Em um deles, cerca de 10\% das entrevistadas foi vítima de violência doméstica ${ }^{(22)} \mathrm{e}$, em outro, $37,7 \%$ das mães estavam sob a guarda de serviços de proteção( ${ }^{(16)}$.

Quanto às substâncias de uso/abuso, os estudos apresentaram uma grande variação de drogas ilícitas, como ecstasy, maconha e cocaína ${ }^{(17)}$; heroína, metadona, cocaína, cannabis, benzodiazepinas e anfetaminas ${ }^{(25)}$; cannabis e/ou anfetaminas ou opiáceos ${ }^{(19-20)}$; crack ou cocaína ${ }^{(23)}$; opiáceos (heroína, metadona, buprenorfina, tramadol, codeína, petidina), estimulantes (cocaína, anfetaminas e seus derivados) e depressores (benzodiazepínicos e álcool) ${ }^{(22)}$; heroína e anfetamina ${ }^{(15)}$; opioides $^{(24)}$, tendendo ao poli consumo e sem descartar o uso de álcool e tabaco concomitantemente. Dois estudos exploraram os efeitos da buprenorfina ou metadona em crianças filhas de grávidas usuárias de drogas e a sua relação com a síndrome de abstinência neonatal|(18,21).

Estudos apontaram também que crianças expostas ao consumo de múltiplas drogas pela mãe durante a gravidez exigiram um período de duração de tratamento mais longo devido a questões clínicas, permanecendo hospitalizadas por mais tempo, em comparação com as crianças expostas a uma única classe de drogas ${ }^{(18,22)}$.

$\mathrm{O}$ número de abortos e mortes neonatais apresentado nos estudos desta revisão foi bastante significativo, levando-se em conta todos os estudos. Um estudo apontou que $7,7 \%$ das crianças necessitaram de manobras de ressuscitação ao nascer devido ao baixo Apgar ${ }^{(25)}$; em um estudo, das 126 entrevistadas, 4 tiveram aborto espontâneo e não puderam participar da pesquisa(17); em outro, das 102 gestantes, 13 abortaram $^{(20)}$; em outro, 5 crianças morreram ${ }^{(23)} ; 1$ bebê nasceu morto e cinco prematuros ao extremo ${ }^{(21)}$; um bebê morreu de síndrome de morte súbita infantil 6 semanas após a alta hospitalar; um bebê foi acidentalmente asfixiado pela mãe na enfermaria pós-natal; outro afogado na banheira em casa com a idade de 18 meses e 2 morreram de complicações de prematuridade extrema ${ }^{(22)}$; em um estudo, houve um natimorto e 2 óbitos neonatais ${ }^{(15)}$. De uma amostra inicial de 450 díades, 6 bebês eram natimortos, da amostra final do mesmo estudo (437), dois bebês morreram logo após o parto, um bebê morreu aos 7 dias por complicações da prematuridade e outro bebê com anomalias múltiplas morreu de insuficiência renal. Uma criança a termo morreu repentinamente em casa com 25 dias. Nesse último estudo, a taxa de morte fetal de $1,3 \%$ foi quase o dobro da verificada no hospital como um todo ${ }^{(18)}$.

Além das mortes neonatais, em um estudo com amostra inicial de 293 mães, três delas vieram a óbito, 
uma por infecção generalizada, ainda no hospital, e duas por overdose após a alta, em casa(22). Também se constatou que as mulheres usuárias de drogas apresentaram histórico de pouco cuidado no período pré-natal obstétrico ${ }^{(15,21)}$.

Nesta revisão, chamou a atenção o número de mães que perderam a guarda de seus filhos e o número de crianças que foram deixadas em orfanatos ou sob a custódia de parentes ${ }^{(15,20,23)}$.

Questões referentes ao aleitamento materno foram discutidas em três estudos, que adotaram posicionamentos contrários quanto à amamentação por mulheres usuárias de drogas. Enquanto em dois deles as mães deveriam ser encorajadas e apoiadas a amamentar independente de continuarem ou não fazendo uso de drogas ${ }^{(15,22)}$, um outro estudo se posicionou contra, argumentando que a amamentação deve ser desencorajada desde o início se as mães fazem uso abusivo, devendo ocorrer apenas se nenhuma droga for detectada na urina e o consumo tiver sido esporádico no início da gravidez ${ }^{(25)}$.

\section{Discussão}

O desenvolvimento de um ser humano é um processo resultante de um emaranhado de situações complexas que envolvem interações recíprocas entre a sua cognição, emoção, fisiologia, percepção do mundo e neurobiologia. O reconhecimento da importância de tais interações para o desenvolvimento humano é o princípio fundamental de modelos que visam nortear pesquisas na perspectiva da ciência do desenvolvimento, como o modelo bioecológico de desenvolvimento humano, que destaca a importância de se pesquisar os processos de interação entre a pessoa e o ambiente, as características da pessoa, as características relativas ao tempo histórico, cultural e social, e por fim, as características do contexto - ambiente físico, social e cultural de uma pessoa para se entender o seu desenvolvimento(26).

Tomando como ponto de partida o olhar através dessa lente, é possível perceber que os efeitos do consumo de drogas parental no desenvolvimento e saúde mental da criança são provenientes de fatores múltiplos, muitos deles anteriores à sua concepção, como o ambiente e estilo de vida adotado por seus progenitores.

Assim, a avaliação do risco quando a gestação é exposta a drogas é difícil, já que os resultados podem ser confundidos pelo consumo concomitante de outros tóxicos ou pela presença de outros fatores socioeconômicos desfavoráveis ${ }^{(12)}$. Além disso, é preciso levar em conta que embora o uso de substâncias psicoativas implique em potencial para o dano em curto ou em longo prazo, os efeitos nocivos mais importantes (efeitos crônicos para a saúde, efeitos biológicos, problemas sociais graves e problemas sociais crônicos) para os usuários resultam da quantidade acumulada da substância psicoativa, do padrão de uso ou da forma ou meio pelo qual ela é utilizada(27)

Diante disso, a elevada prevalência de abuso de substâncias psicoativas na população de mulheres que engravidam expondo o feto aos tóxicos no período de maior vulnerabilidade, particularmente com respeito ao desenvolvimento neurológico e organogênese, configura-se como um sério problema de saúde pública ${ }^{(28)}$ cuja importância foi também evidenciada neste estudo, visto que $81 \%$ dos artigos selecionados foram construídos a partir do binômio mãe-filho, enfocando, principalmente, o período gestacional e puerpério.

Do ponto de vista biológico, os estudos desta revisão revelaram sérias consequências do uso de substâncias psicoativas durante a gestação para o recém-nascido. Como a maioria das drogas utilizadas pelas mães ultrapassam a barreira placentária e hematoencefálica sem metabolização prévia, elas atuam durante o período embrionário, fase de desenvolvimento e formação dos órgãos fetais, causando deficit cognitivos, malformações, síndromes de abstinência, dentre outros ${ }^{(29)}$, como prematuridade, baixo peso ao nascer e diminuição do perímetro cefálico, considerados fatores de alto risco para o desenvolvimento de problemas de desenvolvimento, aprendizagem e adaptação psicossocial da criança, além de problemas de comportamento sugestivos para atendimento psicológico em idade mais avançada ${ }^{(10)}$.

Os resultados de um dos estudos sugerem ainda que os filhos de mulheres que interrompem o uso estão em maior risco para atrasos no desenvolvimento em níveis de exposição mais elevados ${ }^{(17)}$. Em outro estudo, a exposição à cocaína durante o primeiro trimestre previu diminuição do peso e altura aos 7 anos, sendo capazes de compensar os efeitos da exposição à droga somente após essa idade, possivelmente em decorrência da plasticidade no desenvolvimento em crianças nessa faixa etária(23). Em ambos estudos, os efeitos sobre as crianças resultam da exposição à droga no início da gravidez, reforçando a forte influência de fatores biológicos ao longo do desenvolvimento do indivíduo.

Os resultados aqui apresentados são corroborados por pesquisas realizadas em vários estados do

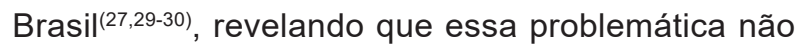
é exclusiva dos países desenvolvidos. Contudo, é importante destacar a influência dos fatores sociais sobre o desenvolvimento da criança. Nessa perspectiva, os efeitos do abuso de drogas sobre o feto podem ser 
facilmente confundidos, influenciados ou desencadeados pela privação social, saúde materna e estilo de vida(18) apresentado por um grande número de mulheres que consomem drogas como aqui evidenciado.

Conforme os estudos demonstraram, ainda é grande o número de grávidas usuárias de drogas com uma assistência pré-natal precária e/ou inexistente, como também é grande o número de abortos e mortes neonatais entre elas. Pesquisas que estudaram o efeito do cuidado pré-natal na mortalidade perinatal, neonatal e infantil apontam que a ausência de cuidado pré-natal e/ou um pré-natal realizado de forma inadequada é fator de risco tão importante para a mortalidade neonatal quanto condições socioeconômicas desfavoráveis das mães, reforçando a necessidade de somar uma atenção pré-natal e pós-parto de qualidade à implementação de melhorias de qualidade de vida, em especial para as gestantes em piores condições socioeconômicas ${ }^{(31)}$.

Uma inter-relação importante entre os aspectos biológicos, sociais e culturais do desenvolvimento humano evidenciados neste estudo foi relacionada ao aleitamento materno. Prova disso, embora um estudo tenha se posicionado contrário à continuidade da amamentação por mulheres em uso de drogas ${ }^{(25)}$, uma vez que essas substâncias passam para o lactente por meio do leite materno variando de acordo com a dose e o padrão de utilização da droga pela mãe ${ }^{(24)}$, a continuidade da amamentação e incentivo ao aleitamento foram mantidos em dois estudos, visto que $\mathrm{o}$ ato de amamentar, além de confortar os bebês ${ }^{(18,22) \text {, }}$ contribui para que eles necessitem de menos cuidados hospitalares ao manifestarem a síndrome de abstinência neonatal ${ }^{(18)}$.

O consumo de drogas parental e efeitos no comportamento e na saúde mental da criança foram investigados por um menor número de estudos ${ }^{(16-17,19,23)}$. Dentre eles, apenas dois incluíram pai e/ou mãe biológicos ou substitutos e professores ${ }^{(19,23)}$.

Associações entre problemas de comportamento e variáveis do ambiente familiar têm sido interesse de várias pesquisas, sendo os eventos de vida negativos provenientes da família apontados como particularmente prejudiciais ao desenvolvimento infantil, configurandose como fator predisponente a problemas de saúde mental e de comportamento ao longo do seu ciclo de crescimento e desenvolvimento, sobretudo quando a criança vivencia algum tipo de violência, seja ela física, psicológica, sexual ou por negligência(32).

Assim, eventos demonstrados nesta revisão, como a morte materna, a perda da guarda dos filhos ${ }^{(15,20,23)} \mathrm{e}$ a violência sofrida pelas mães ${ }^{(16,22)}$ por causas relacionadas ao uso de substâncias psicoativas, são adversidades que podem contribuir para efeitos diferentes sobre a psicopatologia na infância, além de exercerem efeito sobre os transtornos de uso de substâncias em idades mais avançadas ${ }^{(33-34)}$

Estudos sobre a exposição à violência doméstica na infância (ser testemunha, vítima ou ambos) evidenciaram que crianças expostas a tais adversidades estavam mais constantemente em risco para toda a gama de problemas de comportamento, apresentando maiores resultados para delinquência e a depressão em idades mais avançadas ${ }^{(35)}$.

Os resultados de um dos estudos analizados nesta pesquisa evidenciou que as crianças que foram enviadas a orfanatos tiveram menos problemas de comportamento do que as crianças que vivem com suas mães biológicas que continuaram consumindo droga após o nascimento do filho ${ }^{(19)}$, o que pode ser explicado pelo fato de que famílias que funcionam mal podem não ser capazes de fornecer apoio, segurança ou monitoramento essenciais para o desenvolvimento saudável da criança, visto que com tais características um ambiente familiar desregulado, em que a exposição a adversidades estressantes é mais provável, é também um em que os efeitos de tais estressores podem ser menos reconhecidos, reconhecidos ou efetivamente gerenciados $^{(36)}$.

Crianças expostas a drogas intraútero e após seu nascimento tiveram maiores problemas, como o aumento de desatenção, impulsividade e com os pares, além de problemas de comportamento mais significativos, como defict de atenção, baixo desempenho escolar, agressividade, ansiedade e depressão, em comparação com crianças que não foram expostas a drogas ${ }^{(19,23)}$.

Entre os determinantes sociais, o menor apoio social, a baixa escolaridade materna, a baixa qualidade do ambiente do cuidado e a violência urbana evidenciados na maioria dos estudos aqui apresentados também se constituíram como fatores de risco que impactaram negativamente no desenvolvimento e saúde mental das crianças, tendo em vista que as famílias pertencentes a esses estudos viviam em sua maioria em centros urbanos caracterizados por extrema pobreza, baixo índice de escolaridade e alto índice de consumo de drogas.

Diante disso, ressalta-se a importância da detecção precoce dos fatores de risco relacionados ao uso de drogas parental e especialmente pelas gestantes através da realização de consulta de pré-natal humanizada e de qualidade, permitindo o direcionamento correto das medidas necessárias para melhorar a qualidade da gestação tanto para a mãe como para o feto, o que pode contribuir para a diminuição das complicações no recém-nascido ${ }^{(32)}$, bem como o oferecimento de suporte 
social para que essas famílias sintam-se cuidadas e seguras para oferecer o melhor cuidado aos seus filhos.

\section{Conclusões}

O consumo abusivo de drogas parental influencia, sob diversos aspectos, o crescimento e desenvolvimento dos seus filhos, além de poder ocasionar problemas de saúde mental e comportamentais, seja pelas consequências diretas do consumo de substâncias psicoativas, seja pelos efeitos indiretos, como abandono e descuido, falta de carinho e atenção, maus-tratos, entre outros, que tal consumo gera para a criança.

Além disso, os resultados negativos do uso de drogas parental identificados neste estudo incluíram aqueles desencadeados pela exposição intraútero da criança à droga que resultam em alterações perceptíveis imediatamente ao nascimento, como aquelas que afetam as medidas antropométricas (estatura, peso ao nascer, perímetro cefálico); os que impactam diretamente nas condições de saúde, como ocorre nas malformações congênitas, na síndrome da dificuldade respiratória ou na síndrome da abstinência neonatal; e aquelas que afetam o desenvolvimento cognitivo, motor e psicológico das crianças ainda em formação, como as relacionadas às habilidades cognitivas complexas, que só podem ser observadas ao longo do desenvolvimento da criança, ou seja, durante a idade escolar e anos posteriores.

Todavia, embora o consumo parental de drogas tenha exercido seu papel como um importante fator de risco para o desenvolvimento e saúde mental das crianças nos estudos apresentados, ele não pode sozinho ser considerado um determinante unânime dos problemas evidenciados, visto que o desenvolvimento humano é fruto de um processo que envolve fatores como as características pessoais, ambientais e sociais de um indivíduo.

Espera-se contribuir com o conhecimento acerca da temática, fomentando mais discussões sobre as consequências do uso parental no desenvolvimento e saúde mental infantil, assim como mostrar as consequências que esse consumo gera nas crianças, salientando a importância de campanhas educativas para pais e mães visando à prevenção, redução e/ou interrupção deste ato.

\section{Agradecimentos}

As professoras Ana Maria Pimenta de Carvalho e Patrícia Leila dos Santos, pela colaboração na estruturação do artigo.

\section{Referências}

1. Papalia DE, Olds SW. Desenvolvimento Humano. Porto Alegre: Artmed; 2013.

2. Tszesnioski LC, Nobrega KBG, Lima MLLT, Facundes VLD. Construindo a rede de cuidados em saúde mental infantojuvenil: intervenções no território. Ciênc Saúde Coletiva. 2015;20(2):363-70.

3. Toni CGS, Hecavei VA. Relações entre práticas educativas parentais e rendimento acadêmico em crianças. Psico-USF. 2014;19(3):511-21.

4. Lewis AJ, Galbally M, Gannon T, Symeonides C. Early life programming as a target for prevention of child and adolescent mental disorders. BMC Med. 2014;12(33):1-15. 5. Spittle AJ, Thompson DK, Brown NC, Treyvaud K, Cheong JLY, Lee KJ, et al. Neurobehaviour between birth and 40 weeks' gestation in infants born <30 weeks' gestation and parental psychological wellbeing: predictors of brain development and child outcomes. BMC Pediatr. 2014;14(111):1-15.

6. Cabaj JL, McDonald SW, Tough SC. Early childhood risk and resilience factors for behavioural and emotional problems in middle childhood. BMC Pediatrics. 2014;14(166):1-15.

7. Coles CD, Black MM. Introduction to the special issue: impact of prenatal substance exposure on children's health, development, school performance, and risk behavior. $\mathrm{J}$ Pediatr Psychol. 2006;31(1):1-4.

8. Botelho APM, Melo VH, Rocha RC. Uso e dependência de cocaína/crack na gestação, parto e puerpério. Femina. 2013;41(1):23-32.

9. Halpern R, Giugliani ERJ, Victora CG, Barros FC, Horta BL. Fatores de risco para suspeita de atraso no desenvolvimento neuropsicomotor aos 12 meses de vida. J Psiquiatr. 2000;76(6):421-8.

10. Portela GLC, Barros LM, Frota NM, Landim APP, Caetano JÁ, Farias FLR. Percepção da gestante sobre o consumo de drogas ilícitas na gestação. SMAD, Rev. Eletrônica Saúde Mental Álcool Drog. 2013;9(2):58-63.

11. Sithisarn T, Granger DT, Bada HS. Consequences of prenatal substance use. Int J Adolesc Med Health; 2012;24(2):105-12.

12. Ruoti CM, Calabrese E, Ontano M, Airaldi L, Gruhn E, Galeano J, et al. Uso y abuso de drogas durante el embarazo: revisión. Mem Inst Invest Cienc Salud. 2009;5(2):32-44.

13. Soares CB, Hoga LAK, Peduzzi M, Sangaleti C, Yonekura T, Silva DRAD. Integrative review: concepts and methods used in nursing. Rev Esc Enferm USP. 2014;48(2):335-45.

14. Melnyk BM, Fineout-Overholth E. Evidence-Based Practice in Nursing \& Healthcare. Philadelphia: Lippincott Williams \& Wilkins; 2011. 
15. Thaithumyanon $P$, Limpongsanurak S, Praisuwanna P, Punnahitanon S. Perinatal effects of amphetamine and heroin use during pregnancy on the mother and infant. $\mathrm{J}$ Med Assoc Thai. 2005;88(11):1506-13.

16. Lam WKK, Cance JD, Eke AN, Fishbein DH, Hawkins SR, Williams JC. Children of African-American Mothers Who Use Crack Cocaine: Parenting Influences on Youth Substance Use. J Pediatr Psychol. 2007;32(8):877-87.

17. Singer LT, Moore DG, Min MO, Goodwin J, Turner JJ, Fulton S, et al. One-year outcomes of prenatal exposure to MDMA and other recreational drugs. Pediatrics. 2012;130(3):407-13.

18. Dryden C, Young D, Hepburn M, Mactier H. Maternal methadone use in pregnancy: factors associated with the development of neonatal abstinence syndrome and implications for healthcare resources. BJOG. 2009;116:66571.

19. Hjerkinn B, Lindbæk M, Rosvold EO. Behaviour among children of substance-abusing women attending a Special Child Welfare Clinic in Norway, as assessed by Child Behavior Checklist (CBCL). Scand J Caring Sci. 2013;27:285-94.

20. Hjerkinn B, Rosvold EO, Lindbæk M. Neonatal findings among children of substance-abusing women attending a special child welf are clinic in Norway. Scand J Public Health. 2009;37:751-7.

21. Blandthorn J, Forster DA, Love V. Neonatal and maternal outcomes following maternal use of buprenorphine or methadone during pregnancy: findings of a retrospective audit. Women Birth. 2011;24:32-9.

22. Pong KM, Abdel-Latif ME, Lui K, Wodak AD, Feller $\mathrm{JM}$, Campbell T, et al. The temporal influence of a heroin shortage on pregnant drug users and their newborn infants in Sydney, Australia. Austr N Z J Obstet Gynaecol. 2010;50:230-6.

23. Richardson GA, Goldschmidt L, Leech S, Willford J. Prenatal cocaine exposure: Effects on mother- and teacherrated behavior problems and growth in school-age children. Neurotoxicol Teratol. 2011;33(1):69-77.

24. Kelly L, Dooley J, Cromarty H, Minty B, Morgan A, Madden S, et al. Narcotic-exposed neonates in a First Nations population in northwestern Ontario. Can Fam Physician. 2011;57:441-7

25. Gómez SO, López-Vilchez MA, Ledo FD, Ponce EC, Rabasco AC, Collado RC, et al. Consumo de drogas durante la gestación y su repercusión neonatal. Análisis de los perí́odos 1982-1988 y 2002-2008. Med Clin. (Barc). 2011;136(10):423-30.

26. Cerqueira-Silva S, Dessen MA, Costa Júnior A. As contribuições da ciência do desenvolvimento para a psicologia da saúde. Ciênc Saúde Coletiva. 2011;16(1):1599-609.
27. Organização Mundial da Saúde. Neurociência do uso e da dependência de substâncias psicoativas. Genebra: ROCA; 2006.

28. Blasco AM, González ME, Gálvez MM, Lozano BI, Merino GF, Cuenca CF, et al. Exposición a tabaco, alcohol y drogas de abuso en gestantes. Estudio de prevalencia en gestantes de Málaga (España). Adicciones. (Palma de Mallorca). 2015;27(2): 99-108.

29. Cembranelli E, Campos LRF, Portella M, Abreu PVC, Salomão PC, Monteiro DLM. Consequências do uso de cocaína e metanfetamina durante a gravidez. FEMINA. 2012;40(5):241-45.

30. Gallo EAG, Anselmi L, Dumith SC, Scazufca M, Menezes AMB, Hallal PC, et al. Tamanho ao nascer e problemas de saúde mental aos 11 anos em uma coorte brasileira de nascimentos. Cad Saúde Pública. 2011;27(8):1622-32. 31. Li C, Yan H, Zeng L, Dibley MJ, Wang D. Predictors for neonatal death in the rural areas of Shaanxi Province of Northwestern China: a cross-sectional study. BMC Public Health. 2015;15:387.

32. Ellonen N, Piispa M, Peltonen K, Oranen M. Exposure to parental violence and outcomes of child psychosocial adjustment. Violence Vict. 2013;28(1):3-15.

33. Benjet C, Borges G, Medina-Mora ME. Chronic childhood adversity and onset of psychopathology during three life stages: childhood, adolescence and adulthood. Journal Psychiatr Res. 2010;4(11):732-40.

34. Lamela D, Figueiredo B.Coparenting after marital dissolution and children's mental health: a systematic review. J Pediatr. (Rio J). 2016;92(4):331-42.

35. Moylan CA, Herrenkohl TI, Sousa C,Tajima EA, Herrenkohl RC, Russo MJ. The Effects of Child Abuse and Exposure to Domestic Violence on Adolescent Internalizing and Externalizing Behavior Problems. J Fam Violence. 2010;25(1):53-63.

36. Hooven C, Nurius PS, Logan-Greene P, Thompson EA. Childhood Violence Exposure: Cumulative and Specific Effects on Adult Mental Health. Fam Violence. 2012;27(6):511-22. 